\title{
Secretomes of human pluripotent stem cell-derived smooth muscle cell progenitors upregulate extracellular matrix metabolism in the lower urinary tract and vagina
}

\section{Guobing Zhuang}

Stanford University School of Medicine

Yan Wen ( $\sim$ yanwen@stanford.edu )

Stanford University School of Medicine https://orcid.org/0000-0003-4271-3212

Mason Briggs

Stanford University School of Medicine

Qingchun Shao

Stanford University School of Medicine

Darlene Tran

Stanford University School of Medicine

Hongbo Wang

Wuhan Union Hospital

Bertha Chen

Stanford University School of Medicine

\section{Research}

Keywords: secretome, smooth muscle cell progenitors, pluripotent stem cells, iPSC, stress urinary incontinence

Posted Date: October 26th, 2020

DOl: https://doi.org/10.21203/rs.3.rs-95565/v1

License: (c) (1) This work is licensed under a Creative Commons Attribution 4.0 International License. Read Full License

Version of Record: A version of this preprint was published at Stem Cell Research \& Therapy on April 6th, 2021. See the published version at https://doi.org/10.1186/s13287-021-02292-y. 


\section{Abstract}

Background: Adult mesenchymal stem cells (MSCs) have been studied extensively for regenerative medicine, however, they have limited proliferation in vitro, and the long culture time induces cell senescence. MSCs also contribute to tissue repair through their paracrine function. In this study, we sought to examine the paracrine effects of smooth muscle cell progenitors (pSMC) on the urethra and adjacent vagina of stress urinary incontinence rodents. We use human pluripotent stem cell (PSC) lines to derive pSMCs to overcome the issue of decreased proliferation and to obtain a homogenous cell population. This novel approach for treatment of urinary incontinence can also be expanded into treatments for other pelvic floor disorders.

Method: Three human PSC lines were differentiated into pSMCs. The conditioned medium (CM) from pSMC culture, which contain pSMC secretomes, was harvested. To examine the effect of the CM on the extracellular matrix of the lower urinary tract, human bladder smooth muscle cells (bSMCs) and vaginal fibroblasts were treated with pSMC-CM in vitro. Stress urinary incontinence (SUI) was induced in rats by surgical injury of the urethra and adjacent vagina. SUI rats were treated with PSMC-CM and monitored for 5 weeks. Urethral pressure testing was performed prior to euthanasia, and tissues were harvested for PCR, Western Blot and histological staining. Kruskal-Wallis one-way ANOVA test and Student t-test were used for statistical comparisons.

Results: pSMC-CM upregulated MMP-2, TIMP-2, collagen, and elastin gene expression, and MMP-9 activity in human bladder and vaginal cells consistent with elastin metabolism modulation. pSMC-CM treatment restored in vivo urethral function (increase in leak point pressure compared to intact controls, $\mathrm{p}<0.05)$ and increased collagen and elastin expression in the urethra and the adjacent vagina. pSMC-CM also restored the smooth muscle cell layer in the adjacent vagina.

Conclusion: Conditioned media from smooth muscle cell progenitors derived from pluripotent stem cells restored urethral function and vaginal smooth muscle cell and elastin content. These findings support a novel therapeutic potential for PSC-based treatments for SUI and pelvic floor disorders where tissues are affected by elastin and smooth muscle loss.

\section{Background}

Stress urinary incontinence (SUI) is one of the most prevalent pelvic floor disorders affecting many adults worldwide [1]. SUI is defined by an involuntary loss of urine during physical effort and its etiology is thought to be multifactorial[2]. Pregnancy, vaginal delivery, trauma, pelvic surgery, and aging are among the list of factors associated with the development of SUI [3-5]. Histologically, what is observed is a loss of muscular structure and cells in the urethral sphincter with a shift to collagenous tissue, as well as vascular and nerval changes in tissue of the lower urinary tract[6]. Current treatment options include pelvic floor physical therapy [7], bulking agent injections into the urethral tissue, or surgery with placement of synthetic mesh sling around the mid-urethra to provide support[8-10]. Surgical treatment is 
efficacious but the adverse events, including general risks of surgery, synthetic mesh complications, nerve injury, bladder perforation, postoperative voiding dysfunction $[11,12]$ and long term recurrence rates, have led to a quest for the development of new approaches.

Data from animal models using animal autologous mesenchymal stem cells (MSC) transplanted into the urethra suggest that these cells may assist tissue repair through secretion of trophic factors, rather than by significant cell regeneration resulting from in vivo differentiation of the MSC into terminally differentiated muscle cells [13]. Furthermore, several studies on stem cell-derived secretory factors suggest that the secreted factors alone, without presence of the stem cells, may be sufficient to enhance tissue repair after injury. The secreted factors are referred to as secretome, microvesicles, or exosome and can be found in the medium where the stem cells are cultured, also called conditioned medium (CM) [14].

Human-induced pluripotent stem cells (hiPSCs) have also been explored as a stem cell source for treatment of SUI. A unique advantage of these cells over the adult MSCs is that they can be expanded in large quantities and differentiated in vitro into autologous organ-specific cells using defined and reproducible protocols $[15,16]$. Expansion of adult MSCs can be limited by availability, decreased proliferation, and cell senescence. Our previous study indicated that periurethral transplantation of hiPSCderived smooth muscle cell progenitors (pSMCs) promotes functional restoration of the injured rat urethra through pSMC engraftment into the tissues [17]. We also observed significant changes in the native rodent tissue extracellular matrix (ECM) in the PSMC transplanted urethras with a shift to increased elastin deposition rather than the stiff, collagenous tissue typical of chronic injury.

Given mounting data on the anti-fibrotic effects of MSC conditioned media [18], and our observations of in vivo changes in rodent ECM with human PSMC transplantation, we hypothesize that the PSMC conditioned media (pSMC-CM) derived from pluripotent stem cells will also modulate ECM in the urethra and adjacent vagina to restore urethral function in the SUI rodent model. In the present study, we sought to characterize the effect of PSMC-CM on ECM metabolism in human lower urinary tract and vaginal cells in vitro and examine the in vivo regenerative effects of PSMC-CM on the urethra and vagina of the SUI rodent model. These proof of concept studies on the effect of progenitor cell secretomes will expand the therapeutic potential of pluripotent stem cell-based therapies for SUI and for other pelvic floor disorders associated with deficient tissues.

\section{Methods}

\section{Cell Culture}

This study was approved by the Institutional Review Board of the Stanford University School of Medicine and the Stanford University Stem Cell Research Oversight Committee. Three human pluripotent stem cell (PSC) lines were used in this study for differentiation into pSMCs: 1) Huf5-iPSC line was reprogrammed from a $46 y$ o female dermal fibroblasts via viral transduction of the transcription factors 0ct3/4, Sox2, KIf4 and c-Myc [19]; 2) BIR-iPSC line was reprogrammed from a 18yo female dermal fibroblasts with 
modified miRNA reprogramming method [20]. mRNA encoding Oct4, Klf4, Sox2, c-Myc and Lin28 were cotransfected with a mixture of several miRNAs into the fibroblasts to induce iPSCs; and 3) H9 human embryonic stem cell line was a gift from Dr. Joseph Wu, Stanford University. All PSC lines were cultured on Matrigel-coated dishes (BD Biosciences, San Diego, CA) in mTeSR medium (StemCell Technologies, Vancouver, BC).

Human bladder smooth muscle cells (bSMCs) were isolated from three female donors (30, 33 and 50years old which were labeled as B1, B2, B3 respectively). IRB exemption and external approval from Donor Network West's (federally designated organ procurement organization) Internal Research Council and Medical Advisory Board Research Subcommittee was obtained for collection of primary bladder tissue from the three female transplant donors. Patients with history of bladder pathology were excluded from study. The muscular layer of fresh bladders was cut into $2 \mathrm{~mm} \times 2 \mathrm{~mm}$ pieces and cultured in $80 \%$ Dulbecco's modified Eagle's medium (DMEM; Invitrogen, Carlsbad, CA) and 20\% fetal bovine serum (Invitrogen) at $37{ }^{\circ} \mathrm{C}$ in an atmosphere of $95 \%$ air and $5 \% \mathrm{CO}_{2}$. Tissue fragments were removed on day 14 and culture medium was changed to $90 \%$ DMEM and $10 \%$ FBS. Cells were passed to next passage with 0.05\% Trypsin with EDTA (Thermo Scientific, Fremont, CA) when they were 80-90\% confluent. SMC markers including aSMA and SM22 were detected by immunofluorescent staining to confirm cell types (data not shown).

Human vaginal fibroblasts were isolated from three female donors which were labeled as F1(66y), F2(69y), F3(65y) respectively. Fibroblasts cell explants were cut into approximately $1 \mathrm{~mm} \times 1 \mathrm{~mm}$ fragments and cultured in $80 \%$ Dulbecco's modified Eagle's medium (DMEM; Invitrogen) and $20 \%$ fetal bovine serum (Invitrogen) at $37{ }^{\circ} \mathrm{C}$ in an atmosphere of $95 \%$ air and $5 \% \mathrm{CO}_{2}$ [21]. Tissue fragments were removed on day 14 and culture medium was changed into 90\% DMEM and 10\% FBS. Cells were passed to next passage with $0.05 \%$ Trypsin with EDTA (Thermo Scientific, Fremont, CA) when they were 80-90\% confluent.

\section{Directed differentiation of human pluripotent stem cells to pSMCs and conditioned medium collection}

Human pSMCs were differentiated from the three pluripotent stem cell lines using a modified, feeder cellfree, vascular progenitor protocol $[20,22,23]$. Briefly, the stem cells were seeded at a density of 5,00020,000 cells per $\mathrm{cm}^{2}$ of a 10-cm dish precoated with Matrigel in mTeSR supplemented with Thiazovivin $(2 \mu \mathrm{M}$; Cayman Chemical Company, Ann Arbor, MI). After 24-72 h in cultured in chemical defined medium (RPMI 1640 with $1 \mathrm{mM}$ glutamax, 1\% nonessential amino acids, $0.1 \mathrm{mM} \beta$-mercaptoethanol, $1 \%$ penicillin and streptomycin [Life Science Technology, Inc.], 1\% ITS [Corning, Tewksbury, MA]) supplemented with Activin A (50 ng/mL), BMP4 (50 ng/mL; PeproTech, Rocky Hill, NY), and $2.5 \mu \mathrm{M} / \mathrm{mL}$ GSK 3 inhibitor, CHIR99021 on day 0, $2.0 \mu \mathrm{M} / \mathrm{mL}$ CHIR99021 on day 1 (Cayman Chemical Company), followed by basic fibroblast growth factor ( $50 \mathrm{ng} / \mathrm{mL}$ ) and vascular endothelial growth factor ( $40 \mathrm{ng} / \mathrm{mL}$; PeproTech) from day 2 to $12-14$ days. 
The differentiated cells were dissociated with Acutase (Innovative Cell Technologies, San Diego, CA) and prepared for magnetic-activated cell sorting (MACS) and fluorescence-activated cell sorting (FACS) of $\mathrm{CD} 31^{+} / \mathrm{CD} 34^{+}$vascular progenitor cells (VPCs) [24]. Briefly, the cells were first immunolabeled with CD34 microbeads for MACS (Miltenyi Biotec, Auburn, CA), and magnetic labeling was performed according to the manufacturer's instruction. The magnetically labeled CD34+ VPCs were obtained by positive selection. The CD $34^{+}$VPCs sorted by MACS were immediately blocked with mouse IgG (R\&d Systems, Inc., Minneapolis, MN) for 10 min and stained with FITC mouse anti-human CD31 and PerCP-Cy5.5 mouse anti-human CD34 antibodies (BD biosciences) for $30 \mathrm{~min}$ at $4{ }^{\circ} \mathrm{C}$. The CD34+/CD31 + cells were sorted on a FACS Aria II (BD Biosciences). After sorting, the cells which were now labeled as passage 0 (P0), were expanded on mouse collagen IV-precoated plates (BD Biosciences) in smooth muscle cell growth medium (SMGS; Invitrogen) supplemented with recombinant human platelet-derived growth factor-BB (10 ng/mL, PDGF-BB; PeproTech) to yield pSMCs. The pSMCs were passed with $0.05 \%$ Trypsin with EDTA (Thermo Scientific, Fremont, CA) when they reached $80-90 \%$ confluency until they were harvested at passage $3(\mathrm{P} 3)$. Conditioned medium was collected and frozen at $-80^{\circ} \mathrm{C}$ when medium was changed every other day from P0 to P3. The conditioned medium for each cell line was pooled at P0 and at P3, and a small amount of the pooled conditioned medium from each passage was frozen at $-80{ }^{\circ} \mathrm{C}$. The conditioned medium was collected and pooled at P4 for each cell line and concentrated 50 times by ultrafiltration using centrifugal filter units with 10-kDa cutoff (Sartorius Stedim SUS Inc., CA) for rodent periurethral injection.

\section{In vitro treatment of human bladder and vaginal cells with pSMC-CM}

Vaginal fibroblasts and bladder SMCs were passaged onto 6-well plates at passage 2 at a density of 10,000 cells per $\mathrm{cm}^{2}$ in $10 \%$ FBS and $90 \%$ DMEM. When they reached $90 \%$ confluency, medium was changed into basal medium 231 (Invitrogen) supplemented with $0.2 \%$ albumin (Sigma-Aldrich, St. Louis, $\mathrm{MO}$ ) to synchronize for $24 \mathrm{~h}$. The cells were then treated for 48 hours with non-concentrated $\mathrm{CM}$ collected from $\mathrm{PO}$ and $\mathrm{P} 3 \mathrm{pSMCs}$ at $37{ }^{\circ} \mathrm{C}$ in an atmosphere of $95 \%$ air and $5 \% \mathrm{CO}_{2}$, while cells in the control groups were treated with the baseline smooth muscle cell growth medium (SMGS; Invitrogen) supplemented with PDGF preincubated at $37{ }^{\circ} \mathrm{C}$ in an atmosphere of $95 \%$ air and $5 \% \mathrm{CO}_{2}$ for 48 hours.

\section{Animal care and generation of SUI rat model}

Female immunodeficient Rowett Nude rats (RNU, Charles River Laboratories, Hollister, CA, USA, http://www.criver.com/) weighing 200-250 g were used. Animals were maintained at the Stanford University Research Animal Facility in accordance with Stanford University's Institutional Animal Care and Use Committee guidelines. Animal experiments were approved by the Institutional Review Board of the Stanford University School of Medicine and the Stanford Administrative Panel of Laboratory Animal Care (APLAC).

The rat model of SUI was established via transabdominal urethrolysis as described by Rodriguez et al. [25]. This SUI rat model showed significantly decreased urethral resistance (by leak point pressure 
measurements) and urethral smooth muscle damage for at least 8 weeks after surgery $[17,26]$. Bilateral ovariectomy was done on the rodents to eliminate the influence of estrus cycle on the ECM metabolism and to simulate an estrogen-deficiency state of menopause [27]. In brief, RNU rats were intraperitoneally anesthetized with ketamine ( $30 \mathrm{mg} / \mathrm{kg}$ ) and xylazine $(3 \mathrm{mg} / \mathrm{kg})$. The ovaries were exteriorized through a lower abdominal incision. After ovarian vessels were ligated, bilateral ovaries were excised. The bladder and urethra were identified and circumferentially separated from anterior vaginal wall and pubic bone by sharp dissection, thus causing injury to the urethral sphincter and adjacent vagina.

\section{Conditioned media peri-urethral injection and tissue collection}

The rats were randomly divided into four treatment groups: 1 . urethrolysis plus $50 x$ concentrated SMGS (sham-SMGS group, $n=7$ ), 2. urethrolysis plus 50x concentrated H9-pSMC-CM (H9 CM group, $n=6$ ), 3 . urethrolysis plus 50x concentrated Huf5-pSMC-CM (Huf5 CM group, $n=7$ ), 4. urethrolysis plus 50x concentrated BIR pSMC-CM (BIR CM group, $n=8$ ). Three weeks after urethrolysis, rats were anesthetized with $3-4 \% \mathrm{v} / \mathrm{v}$ isoflurane and $100 \mu \mathrm{L}$ of the concentrated pSMC-CM or SMGS were injected in the periurethral area (once weekly for three weeks) at two sites using a 28.5-gauge insulin syringe. Researchers were blinded to the treatment group allocations. Leak point pressure (LPP) testing to evaluate urethral function was performed 5 weeks after initial injection. The rats were then euthanized and urethra and adjacent vagina were harvested. The proximal part of the urethra and vagina was embedded in TissueTek O.C.T. compound (Sakura Finetek, Tokyo, Japan, http://www.sakura-finetek.com) for histologic study. The middle part of the urethra and vagina was used for RNA extraction, and the distal part of the urethra and vagina was used for protein extraction.

\section{Leak point pressure (LPP) measurement}

The LPP measurement was used to assess urethral sphincter function. LPP was performed as described by Conway et al. [28]. Investigators performing LPP measurement were blinded to the group assignment of each animal. Briefly, 5 weeks after the injection, the rats were anesthetized with ketamine $(30 \mathrm{mg} / \mathrm{kg})$ and xylazine $(3 \mathrm{mg} / \mathrm{kg})$. A transvesical catheter with a fire-flared tip was inserted into the bladder dome through a small abdominal incision. The abdominal wall was closed, and the catheter was connected via a three-way stopcock to a 50-ml syringe for filling with methylene blue colored saline and to a pressure transducer (TSD 104A, BIOPAC Systems Inc., CA, USA, http://www.biopac.com) for monitoring bladder pressure. The bladder pressure was amplified and sampled by a biological signal acquisition system (BIOPAC MP 150) and digitalized for computer data collection using Acknowledge acquisition and analysis software (BIOPAC Systems Inc.).

Before LPP testing, the spinal cord was transected at the T8-T10 level to eliminate the voiding reflex mediated by spino-bulbo-spinal pathways. The urethral closure mechanism during urine storage remains intact because urethral contractile reflexes activated by sympathetic and somatic nerves responding to bladder distension are predominantly organized at the lumbosacral spinal cord level. The vertical tilt table/intravesical pressure clamp model was used to measure the LPP. The rat was taped to a board and 
placed in the vertical position. The $50 \mathrm{ml}$ syringe (reservoir) which was connected to the bladder catheter via the three-way stopcock was then fixed onto a metered vertical pole. Bladder filling was done by manually raising the height of the reservoir by $2-3 \mathrm{~cm}$ increments for every 2 minutes starting from $0 \mathrm{~cm}$, until urinary leakage (methylene blue saline) was observed at the urethral meatus. The bladder pressure (measured by the transducer) at which leakage was observed was recorded as the LPP. LPP is thus a measure of the urethral sphincter pressure against bladder filling. The mean of at least three consecutive LPPs was taken as a data point for each animal.

\section{RNA extraction and quantitative reverse transcription- polymerase chain reaction}

Total RNA of cells and rat tissue was extracted with the RNA-STAT-60 reagent (Tel-Test, Inc., Friendswood, TX, USA). RNA yield was determined using a Nanodrop 2000 spectrophotometer (Thermo Scientific). Total RNA $(1 \mu \mathrm{g})$ was reverse transcribed into CDNA using the M-MLV reverse transcriptase system (Thermo Scientific). PCR primers were described previously [17, 24], except human TIMP2 (Sense: AAGCGGTCAGTGAGAAGGAA; Anti-sense: GATGTTCAAAGGGCCTGAGA), rat MMP2 (Sense: GTAAAGTATGGGAACGCTGATGGC; Anti-sense: CTTCTCAAAGTTGTACGTGGTGGA) and rat TIMP2 (Sense: ACACGCTTAGCATCACCCAGAA; Anti-sense: CAGTCCATCCAGAGGCACTCAT). Real-time quantitative reverse transcription-polymerase chain reaction (qRT-PCR) was carried out on the Mx3005P Multiplex Quantitative PCR System with MxPro QPCR software (Stratagene, La Jolla, CA, US). Brilliant SYBR Green QPCR Master Mix (Stratagene) was used to perform PCR. GAPDH was used as an endogenous reference against which the different template values were normalized. All PCR reactions were performed in duplicate. The cycle of threshold (Ct) method was used for quantification. Data were analyzed by MxPro QPCR software.

\section{Western blot assay}

Cell culture supernatant was collected and further concentrated (10x) by ultrafiltration using centrifugal filter units with 10-kDa cutoff (Sartorius Stedim SUS Inc., CA). Human bSMCs or vaginal fibroblasts were washed twice with cold PBS and homogenized on ice with a RIPA buffer (50 mM Tris, $150 \mathrm{mM} \mathrm{NaCl}, 1 \%$ NP40, 0.5\% deoxycholate, 0.1\% SDS, 4 mM EDTA, and 2 mM PMSF, PH 7.4) supplemented with proteinase inhibitor cocktail (Roche Diagnostics $\mathrm{GmbH}$, Basel, Switzerland), and then rotated at $4{ }^{\circ} \mathrm{C}$ for 2 days to solubilize the protein more efficiently. Cell debris was removed by centrifugation at 14,000 RPM for $30 \mathrm{~min}$. Protein extraction from rodent tissue was performed[29]. Total protein concentrations of concentrated supernatants and cell lysates were determined using the Bradford method (Bio-Rad, Hercules, CA). The samples were reduced with a sodium dodecyl sulfate (SDS) sample buffer containing $5 \%$ of 2 -mercaptoethanol and boiled for $7 \mathrm{~min}$. The proteins $(20 \mu \mathrm{g} / \mathrm{lane}$ for cell lysates and rat tissue lysates, $100 \mu \mathrm{g} /$ lane for concentrated supernatants) were subjected to $8-10 \%$ (wt/vol) polyacrylamide gels (SDS-PAGE). The gels were blotted onto nitrocellulose membranes (Bio-Rad) in an electrophoretic transfer cell (Bio-Rad). Blots were blocked with $5 \%$ nonfat milk at $4{ }^{\circ} \mathrm{C}$ overnight, and then probed with mouse anti-TIMP-1 antibody (1:1000; Calbiochem, La Jolla, CA), mouse anti-TIMP-2 antibody (1:200; Calbiochem, La Jolla, CA), rabbit anti-collagen III antibody (1:1000; Abcam, Cambridge, MA), goat anti- 
alpha elastin antibody (1:1000; Abcam, Cambridge, MA), rabbit anti-human elastin antiserum (1:200; Elastin products company, Owensville, MO) at room temperature for $1 \mathrm{~h}$. After washing three times with phosphate-buffered saline with $0.1 \%$ Tween-20, pH 7.4 (PBS-T), the membrane was then incubated with sheep anti-mouse IgG conjugated to HRP (1:5000, GE Healthcare, Pittsburgh, PA) or donkey anti-rabbit IgG conjugated to HRP (1:5000, GE Healthcare) or mouse anti-goat IgG conjugated to HRP (1:5000, GE Healthcare) for $1 \mathrm{~h}$ at room temperature, followed by three washes in PBS-T. Blots were developed by chemiluminescence. The blots were re-probed with goat anti-GAPDH polyclonal antibody (1:5000, Abcam) and then dilution of mouse anti-goat IgG conjugated to HRP (1:5000; Invitrogen). The band density was determined by Image Studio Software (LI-COR, Inc., Lincoln, Nebraska USA).

\section{Elastin staining and qualitative examination of elastin morphology}

Rodent tissues were embedded in OCT compound on liquid nitrogen and stored at $-80{ }^{\circ} \mathrm{C}$ until cryosectioned. The cryosections were cut and mounted on superfrost slides (Thermo Fisher Scientific Life Sciences). The sections were warmed up for 15 minutes to room temperature before fixing in $4 \% \mathrm{wt} / \mathrm{vol}$ cold paraformaldehyde (Thermo Fisher Scientific Life Sciences) in PBS (pH 7.4) at room temperature for 15 minutes. The sections were washed three times in fresh PBS (5minutes per wash) to remove OCT and then rinsed in water once. The elastin fibers (black) were stained in Weigert's Resorcin-Fuchsin solution for 2-4 hours according to the manufacturer's instruction (Electron Microscope Sciences, Hatfield, PA, http://www.electronmicroscopy-sciences.com). The excess solution was removed with $95 \% \mathrm{vol} / \mathrm{vol}$ ethanol. The slides were differentiated with $1 \% \mathrm{vol} / \mathrm{vol}$ acid alcohol and then wash in water. Cell nuclei (dark blue) were stained with Weigert's iron hematoxylin working solution (Poly Scientific R\&D Corporation, Bay Shore, NY, http://www.polyrnd.com) for approximately 30 seconds. The slides were washed well in running water and then counterstained with van Gieson's solution for 3-5 minutes for collagen fibers (red pink). The excess stain was rinsed from the slides with distilled water.

The slides were visually scored by four people separately for elastin length $(1=$ short, $2=$ moderate, $3=$ long), thickness ( 1 = thin, $2=$ moderate, $3=$ thick) and density ( $1=$ sparce, $2=$ moderate density, $3=$ dense), collagen density ( $1=$ sparce, 2 = moderate density, $3=$ dense). The scorers were blinded to the group assignments. Scores were totaled for each characteristic and used for comparison to assess whether there were gross, visible differences in the morphologic characteristics.

\section{Gelatin zymography}

Gelatinolytic activities of matrix metalloproteinases (MMPs) in cell culture supernatants and in cell lysates were assessed by gelatin zymography [30]. In brief, samples were mixed with nonreducing sample buffer before being electrophoresed in $8 \%$ polyacrylamide gels containing $0.1 \%$ gelatin in the presence of SDS. After electrophoresis, the gels were washed twice with $2.5 \%$ Triton X-100 and were subsequently incubated overnight at $37^{\circ} \mathrm{C}$ in the substrate buffer (containing $50 \mathrm{mM}$ Tris-HCL, pH 8, $5 \mathrm{mM}, \mathrm{CaCl}_{2}$, $0.02 \%$ Azide). After staining with Coomassie blue, enzyme activity appeared as clear bands against the blue-stained background. The area of lysis for each band detected was analyzed using Bio-Rad Quality 
One Software (Bio-Rad). The bands density was determined by ImageJ Software (National Institutes of Health, Bethesda, MD, USA).

\section{Statistical Analysis}

Statistical analyses were performed using SPSS version 21 (SPSS Inc., Chicago, IL). Results are expressed as mean \pm SEM. Kruskal-Wallis one-way ANOVA test was used to compare multiple variables. The Student t-test was used for comparison between groups for the in vivo study. A value of $p<0.05$ was considered significant.

\section{Results}

pSMC-CM upregulated extracellular matrix elastin metabolism in human bladder smooth muscle cells (bSMCs) and vaginal fibroblasts

To examine how pSMC- CM regulates ECM metabolism in human bladder smooth muscle cells (B1, B2, B3) and vaginal fibroblasts (F1, F2, F3), qPCR was performed on the cells after treatment with pSMC-CM (Fig. 1). Cells from three participants were treated with PSMC-CM derived from two human PSC lines (Huf5 and BIR). Compared to the cells treated with control medium (SMGS supplemented with PDGF, i.e. medium only without the secretomes), 9/12 groups (B1 + Huf5-CM, B2 + BIR-CM, B3 + Huf5-CM, B3 + BIR$\mathrm{CM}, \mathrm{F} 1+$ Huf5-CM, F1 + BIR-CM, F2 + Huf5-CM, F2 + BIR-CM, F3 + Huf5-CM) treated with pSMC-CM showed a significant increase in TIMP-2 and MMP-2 mRNA expression $(p<0.05)$ (Fig. 1A, 1B). TIMP-1 mRNA expression in bSMCs was not significantly different compared to control medium for all Huf 5 and BIR pSMC-CM; while in 4/6 fibroblasts groups (F1 + BIR-CM, F2 + Huf5-CM, F2 + BIR-CM, F3 + BIR-CM), mRNA expression of TIMP-1 was significantly increased by P3-pSMC-CM $(p<0.05)$ (Fig. 1C).

Western Blot was performed to examine TIMP-1 and TIMP-2 secreted by cells in the supernatant (Fig. 2). In 4/6 bSMCs and in 2/6 fibroblast groups, P3 pSMC-CM treated cells secreted more TIMP-2 than the control medium treated cells $(p<0.05)$. Neither bSMCs nor fibroblasts appear to secrete TIMP-1 when cultured with passage 0 or passage 3 pSMC-CM (Fig. 2A, 2B).

The activities of MMPs in concentrated cell culture supernatants were examined by nonreducing gelatin zymography (Fig. 3). In 8/12 groups, passage 3 pSMC-CM significantly upregulated pro-MMP-9 activity in the supernatant of the treated cells $(p<0.05)$. MMP-2 activity was similar to controls in all groups (Fig. 3 ).

\section{pSMC-CM promoted gene expression of collagen and elastin in bSMCs and vaginal fibroblasts}

Similar to the ECM metabolism protease data reported above, passage 3 pSMC-CM significantly increased gene expression of collagen I, collagen III, and elastin in 9/12 groups (Fig. 4). However, upregulation of collagen I, collagen III, and elastin protein expression was only observed for collagen III in B3 + BIR-CM, F1 + Huf5-CM, and F1 + BIR-CM (data not shown). 


\section{pSMC-CM restored urethral function in a stress urinary incontinence rat model}

Stress urinary incontinence (SUI) was induced in rats by surgical urethrolysis. Leak point pressure of the urethra (LPP) was used to evaluate in vivo urethral function after peri-urethral injection of the SUI rats with PSMC-CM derived from three human pluripotent stem lines: two iPSC lines (Huf5, BIR) and one embryonic stem cell line (H9). Compared with pure control rats (no surgery and no pSMC-CM treatment), mean LPP was significantly lower in the SUI rats treated SMGS (sham controls) 8 weeks after urethrolysis (17.59 $\pm 3.18 \mathrm{~cm} \mathrm{H}_{2} \mathrm{O}$ vs. $\left.36.51 \pm 9.58 \mathrm{~cm} \mathrm{H}_{2} \mathrm{O}, \mathrm{p}<0.05\right)$, indicative of persistent decreased urethral function in the sham controls. Two out of three SUI rat groups treated with pSMC-CM (H9-pSMC-CM and BIR-pSMC-CM) showed significantly higher LPP compared to sham-SMGS rats $\left(26.5 \pm 6.85 \mathrm{~cm} \mathrm{H}_{2} \mathrm{O}\right.$ vs. $17.59 \pm 3.18 \mathrm{~cm} \mathrm{H}_{2} \mathrm{O}, 27.5 \pm 9.50 \mathrm{~cm} \mathrm{H}_{2} \mathrm{O}$ vs. $17.59 \pm 3.18 \mathrm{~cm} \mathrm{H}_{2} \mathrm{O}$ ), demonstrating that pSMC-CM facilitated recovery of urethral function (Fig. 5).

\section{pSMC-CM induced ECM remodeling in the rat urethra}

Our previous study documented that alteration of ECM components in the lower urinary tract after urethrolysis contribute to the development of urine incontinence in SUI rats [17]. To evaluate the effect of pSMC-CM on in vivo ECM remodeling in the lower urinary tract, we examined expression of collagen I, collagen III, elastin, TIMP-2 and MMP-2 in rat urethra and vagina. PSMC-CM treated rats demonstrated increased collagen I, collagen III and TIMP-2 mRNA expression in the BIR pSMC-CM compared to shamSMGS group (Fig. 6A). This was reflected in an increased expression of collagen III and elastin protein in the urethra (Fig. 6B), suggesting that injection of pSMC-CM induced ECM remodeling.

Elastin morphology of pSMC-CM treated rat urethra was assessed by visual scoring done by 4 observers blinded to the study groups. Compared with sham-SMGS, elastic fibers in urethras of the rats treated with H9-pSMC-CM and BIR-pSMC-CM were observed to be thicker, longer and denser. Rats from H9-pSMC-CM and BIR-pSMC-CM group also had denser collagen content in the urethra compared to the sham-SMGS group (data not shown).

pSMC-CM induced ECM remodeling and restoration of the smooth muscle cell layer in SUI rat vagina

Vaginal prolapse is frequently associated with SUI [31], it is thought that the connective tissue and vaginal wall under the urethra provide support and contribute to the urinary continence mechanism. Therefore, we examined ECM remodeling in the rat vagina which is in close proximity to the injection site. H9-pSMC-CM and BIR-pSMC-CM treated SUI rat vaginas had significantly higher gene expression of collagen I, collagen III and elastin compared to the SUI rat vaginas from the sham-SMGS group. These findings agree with the urethral function LPP data presented above. Gene expression of MMP-2 in the vagina was found to be significantly increased in Huf5-pSMC-CM and BIR-pSMC-CM groups compared with the sham-SMGS group (Fig. 7A). 
Collagen III and elastin protein expression in vagina was also examined. Collagen III expression was found to be significantly higher in Huf5-pSMC-CM and BIR-pSMC-CM treated rats, and elastin expression was found significantly higher in H9-pSMC-CM and BIR-pSMC-CM treated rats compared with shamSMGS treated rats (Fig. 7B).

Three samples of vaginal wall tissue were randomly selected from each group and stained by immunofluorescence for smoothelin (a smooth muscle cell protein) to assess whether the injected pSMCCM induced a myogenic response. Rats in the sham-SMGS group exhibited disrupted and thinner smooth muscle layer compared with the pure control group (no surgery or treatment), consistent with smooth muscle cell destruction due to the surgical urethrolysis. Higher smoothelin expression was observed in the pSMC-CM treated groups, suggesting a smooth muscle regeneration effect from the injected CM (Fig. 8).

\section{Discussion}

SUI is thought to arise from the decreased support of the pelvic floor and vaginal connective tissue around the bladder neck and urethra [31], and loss of urethral sphincter muscle [32]. Current surgical treatment is efficacious but the adverse events associated with surgery and permanent synthetic meshes implants, postoperative voiding dysfunction [11, 12], and long-term recurrence rates have led to a quest for the development of new approaches.

Several studies have suggested that a key mechanism by which transplanted adult MSCs contribute to tissue repair is through their paracrine function [33-36]. Our studies using smooth muscle cell progenitors (pSMC) differentiated from human iPSCs showed that pSMC transplantation into the urethra of the SUI rat model also yielded histologic evidence of extracellular remodeling in the native rodent tissue $[17,37]$.

While these data support an in vivo paracrine effect of pSMCs in the SUl rodent model, the long term, in vivo effects of secretomes from PSC-derived progenitor cells have never been tested. In the present study, we sought to further investigate the paracrine effect of human smooth muscle cell progenitors (pSMCs) on the extracellular matrix metabolism and test whether administration of pSMC conditioned media alone, without the cells, can restore urethral function.

Conditioned media from passaging of pSMCs, which contains the cell culture medium and the cell secretomes, were used to evaluate the in vitro effect of pSMCs on cells from the bladder and the vagina. Our data show that in bSMCs, pSMC-CM upregulated gene expression of extracellular matrix proteins MMP-2 and TIMP-2, while it did not affect TIMP-1 gene expression. In vaginal fibroblasts, pSMC-CM upregulated gene expression of MMP-2, TIMP-1, and TIMP-2. Enzyme activity assay revealed that MMP-9 activity was increased with no significant change in MMP-2 activity in cells treated with PSMC-CM compared to controls. Taken together, these in vitro alterations induced by PSMC-CM treatment suggest modulation of elastin in the ECM, as MMP-2, MMP-9, TIMP-2 are proteases and anti-proteases involved in elastin metabolism. Moreover, gene expression of the main structural components of the ECM (collagen I, 
collagen III, and elastin) which contribute to the biomechanical properties of lower urinary tract soft tissue, were all found to be upregulated in the cells after treatment with PSMC-CM. We believe that the lack of increased collagen and elastin protein expression is due to the short treatment period (48 $\mathrm{h}$ ) which may not enough time for synthesis of more complex proteins.

We used an established SUI rat model to test whether PSMC-CM would result in restoration of urethral function. The in vivo data showed significant functional restoration with PSMC-CM treatment. The SUI rats injected with PSMC-CM showed ECM remodeling with higher local elastin and collagen expression. Interestingly, the smooth muscle layers in the treated vagina adjacent to the urethra was thickened compared to sham treatment. These histologic findings correlated with functional improvement.

Taken together, data from this present study suggest that PSMC-CM contributes to the therapeutic effect of the iPSC-derived smooth muscle cell progenitors and may be sufficient for a therapeutic effect when used alone without the cells.

Limitations of this study include limited sample size and limited ability to detect proteins present in low concentrations. The small samples also precluded our ability to examine a wider range of proteins. We acknowledge that there are likely other growth factors such as transfer growth factors- $\beta$ (TGF- $\beta$ ), vascular endothelial derived growth factor (VEGF), platelet derived growth factor (PDGF), and epidermal growth factor (EGF) that may be involved but were not detected by our assays.

To our knowledge, this is the first study to document that CM from smooth muscle cell progenitors derived from human PSC lines can exert a functional and histological effect on the urethra and vagina. These data support future development efforts for iPSC as stem cell source for progenitor cell-CM therapies for treatment of pelvic floor disorder. This novel treatment would bypass many of the regulatory safety issues associated with cell therapy. Additionally, a major advantage of this approach over using an adult MSC source is the ability to differentiate large homogenous progenitor cell populations for reproducible $\mathrm{CM}$ production. The $\mathrm{CM}$ can be produced and stored in quantities sufficient for multi-dose applications to improve efficacy over time or for prevention of tissue deterioration in pelvic floor disorders.

\section{Conclusion}

Conditioned media from smooth muscle cell progenitors derived from human pluripotent stem cells facilitates restoration of urethral function in vivo. This is accompanied by ECM remodeling through upregulation of MMP-2, MMP-9, TIMP2, and collagen I, collagen III and elastin expression in cells (in vitro) and tissue (in vivo) from the urethra and adjacent vagina. These findings support a novel therapeutic potential for PSC-based treatments for pelvic floor disorders where tissues are affected by elastin and smooth muscle loss.

\section{Abbreviations}


pSMC: Smooth muscle cell progenitors; iPSC:Induced pluripotent stem cells; hESC:Human embryonic stem cell; MSC:Mesenchymal stem cell; ECM:Extracellular matrix; SUI:Stress urinary incontinence; pSMC$\mathrm{CM}$ :Smooth muscle cell progenitors conditioned media; bSMC:Bladder smooth muscle cell; MMP:Matrix metalloproteinase; TIMP:Tissue inhibitor of metalloproteinase; bFGF:Basic fibroblast growth factor; VEGF:Vascular endothelial growth factor; PBST:Phosphate-buffered saline with 0.1\% Tween-20; DMEM:Dulbecco's modified Eagle's medium; CDM:Chemical defined medium; MACS:Magnetic-activated cell sorting; FACS:Fluorescence-activated cell sorting; VPCs:Vascular progenitor cells; SMGS:Smooth muscle cell growth medium; LPP:Leak point pressure; qRT-PCR:Quantitative reverse transcriptionpolymerase chain reaction; PDGF:Platelet derived growth factor

\section{Declarations}

\section{Acknowledgements}

We wish to thank Donor Network West and all of the organ and tissue donors and their families, for giving the gift of life and the gift of knowledge, by their generous donation.

\section{Funding}

California Institute for Regenerative Medicine (CIRM, TRAN1-10958, PI: B. Chen) and the Scientific Research Training Program for Young Talents, Wuhan Union Hospital, China.

\section{Availability of data and materials}

The datasets used and/or analyzed during the current study are available from the corresponding author on reasonable request.

\section{Authors' contributions}

G. Zhuang and Y. Wen: conception and design, collection and/or assembly of data, data analysis and interpretation, manuscript writing; M. B. and Q. Shao and D. Tran: collection and/or assembly of data; H. W.: conception and design; B. C.: conception and design, data analysis and interpretation, manuscript writing, final approval of the manuscript.

\section{Competing interests}

The authors declare that they have no competing interests.

\section{Consent for publication}

Not applicable.

\section{Ethics approval and consent to participate}


This study was approved by the Institutional Review Board of the Stanford University School of Medicine and the Stanford University Stem Cell Research Oversight Committee. All experimental methods were carried out in accordance with the approved guidelines.

\section{Authordetails}

${ }^{1}$ Department of Obstetrics/Gynecology, Stanford University School of Medicine, 300 Pasteur Drive HH333, Stanford, CA 94305, USA (in which the work was done)

${ }^{2}$ Department of Obstetrics/Gynecology, Union Hospital, Tongji Medical Collagen, Huazhong University of Science and Technology, Wuhan, People's Republic of China

\section{References}

1. Norton P, Brubaker L. Urinary incontinence in women. Lancet. 2006;367:57-67.

2. Abrams P, Cardozo L, Fall M, Griffiths D, Rosier P, Ulmsten U, van Kerrebroeck P, Victor A, Wein A. The standardisation of terminology of lower urinary tract function: report from the Standardisation Subcommittee of the International Continence Society. Am J Obstet Gynecol. 2002;187:116-26.

3. Parrillo L, Wein A, Postradical Prostatectomy Incontinence, Prostate Cancer: Science and Clinical Practice, 2nd Edition, (2016) 281-293.

4. Aicher WK, Hart ML, Stallkamp J, Klunder M, Ederer M, Sawodny O, Vaegler M, Amend B, Sievert KD, Stenzl A. Towards a Treatment of Stress Urinary Incontinence: Application of Mesenchymal Stromal Cells for Regeneration of the Sphincter Muscle. Journal of Clinical Medicine. 2014;3:197-215.

5. Danforth KN, Townsend MK, Lifford K, Curhan GC, Resnick NM, Grodstein F. Risk factors for urinary incontinence among middle-aged women. Am J Obstet Gynecol. 2006;194:339-45.

6. Markland AD, Goode PS, Redden DT, Borrud LG, Burgio KL. Prevalence of Urinary Incontinence in Men: Results From the National Health and Nutrition Examination Survey. J Urol. 2010;184:1022-7.

7. Holroyd-Leduc JM, Steinke V, Elliott D, Mullin K, Elder K, Callender S, Hildebrand KA. Improving the Quality of Care for Older Adults Using Evidence-Informed Clinical Care Pathways. Canadian Geriatrics Journal. 2013;16:111-3.

8. Hart ML, Izeta A, Herrera-Imbroda B, Amend B, Brinchmann JE. Cell Therapy for Stress Urinary Incontinence. Tissue Engineering Part B-Reviews. 2015;21:365-76.

9. Sathianathen NJ, McGuigan SM, Moon DA. Outcomes of artificial urinary sphincter implantation in the irradiated patient. Bju International. 2014;113:636-41.

10. Wang HJ, Chuang YC, Chancellor MB. Development of cellular therapy for the treatment of stress urinary incontinence. Int Urogynecol J. 2011;22:1075-83.

11. Fusco F, Abdel-Fattah M, Chapple CR, Creta M, La Falce S, Waltregny D, Novara G. Updated Systematic Review and Meta-analysis of the Comparative Data on Colposuspensions, Pubovaginal 
Slings, and Midurethral Tapes in the Surgical Treatment of Female Stress Urinary Incontinence. Eur Urol. 2017;72:567-91.

12. Ford AA, Rogerson L, Cody JD, Aluko P, Ogah JA. Mid-urethral sling operations for stress urinary incontinence in women. Cochrane Database Syst Rev. 2017;7:CD006375.

13. Deng KL, Lin DL, Hanzlicek B, Balog B, Penn MS, Kiedrowski MJ, Hu ZQ, Ye ZQ, Zhu H, Damaser MS. Mesenchymal stem cells and their secretome partially restore nerve and urethral function in a dual muscle and nerve injury stress urinary incontinence model. American Journal of Physiology-Renal Physiology. 2015;308:F92-100.

14. Kim HO, Choi SM, Kim HS. Mesenchymal Stem Cell-Derived Secretome and Microvesicles as a CellFree Therapeutics for Neurodegenerative Disorders. Tissue Engineering Regenerative Medicine. 2013;10:93-101.

15. Reardon S, Cyranoski D. Japan stem-cell trial stirs envy. Nature. 2014;513:287-8.

16. Fox IJ, Daley GQ, Goldman SA, Huard J, Kamp TJ, Trucco M. Use of differentiated pluripotent stem cells in replacement therapy for treating disease. Science. 2014;345:889-+.

17. Li YH, Wen Y, Wang Z, Wei Y, Wani P, Green M, Swaminathan G, Ramamurthi A, Pera RR, Chen B. Smooth Muscle Progenitor Cells Derived From Human Pluripotent Stem Cells Induce Histologic Changes in Injured Urethral Sphincter. Stem Cells Translational Medicine. 2016;5:1719-29.

18. Harrell CR, Fellabaum C, Jovicic N, Djonov V, Arsenijevic N, Volarevic V. Molecular Mechanisms Responsible for Therapeutic Potential of Mesenchymal Stem Cell-Derived Secretome, Cells, 8 (2019).

19. Byrne JA, Nguyen HN, Pera RAR. Enhanced Generation of Induced Pluripotent Stem Cells from a Subpopulation of Human Fibroblasts, Plos One, 4 (2009).

20. Zhou YY, Kang GGN, Wen Y, Briggs M, Sebastiano V, Pederson R, Chen B. Do Induced Pluripotent Stem Cell Characteristics Correlate with Efficient In Vitro Smooth Muscle Cell Differentiation? A Comparison of Three Patient-Derived Induced Pluripotent Stem Cell Lines. Stem Cells Development. 2018;27:1438-48.

21. Chen $B$, Wen $Y$, Wang $H$, Polan ML. Differences in estrogen modulation of tissue inhibitor of matrix metalloproteinase-1 and matrix metalloproteinase-1 expression in cultured fibroblasts from continent and incontinent women. Am J Obstet Gynecol. 2003;189:59-65.

22. Li YH, Green M, Wen Y, Wei Y, Wani P, Wang Z, Pera RR, Chen B. Efficacy and Safety of ImmunoMagnetically Sorted Smooth Muscle Progenitor Cells Derived from Human-Induced Pluripotent Stem Cells for Restoring Urethral Sphincter Function. Stem Cells Translational Medicine. 2017;6:1158-67.

23. Wang Z, Wen Y, Li YH, Wei Y, Green M, Wani P, Zhang PB, Pera RR, Chen B. Precursor of Smooth Muscle Cells Derived from Human Pluripotent Stem Cells for Treatment of Stress Urinary Incontinence. Neurourol Urodyn. 2016;35:20-0.

24. Li YH, Wen Y, Green M, Cabral EK, Wani P, Zhang F, Wei Y, Baer TM, Chen B. Cell sex affects extracellular matrix protein expression and proliferation of smooth muscle progenitor cells derived from human pluripotent stem cells, Stem Cell Research \& Therapy, 8 (2017). 
25. Rodriguez LV, Chen S, Jack GS, de Almeida F, Lee KW, Zhang R. New objective measures to quantify stress urinary incontinence in a novel durable animal model of intrinsic sphincter deficiency. Am $\mathrm{J}$ Physiol Regul Integr Comp Physiol. 2005;288:R1332-8.

26. Rodriguez LV. Evaluation of different techniques to create chronic urinary incontinence in the rat COMMENT. Bju International. 2009;103:785-6.

27. Kitajima Y, Doi H, Ono Y, Urata Y, Goto S, Kitajima M, Miura K, Li TS, Masuzaki H. Estrogen deficiency heterogeneously affects tissue specific stem cells in mice, Scientific Reports, 5 (2015).

28. Conway DA, Kamo I, Yoshimura N, Chancellor MB, Cannon TW. Comparison of leak point pressure methods in an animal model of stress urinary incontinence. Int Urogynecol J Pelvic Floor Dysfunct. 2005;16:359-63.

29. Wen Y, Ho JYP, Polan ML, Chen B. Expression of Apoptotic Factors in Vaginal Tissues From Women With Urogenital Prolapse. Neurourol Urodyn. 2011;30:1627-32.

30. Toth M, Sohail A, Fridman R. Assessment of gelatinases (MMP-2 and MMP-9) by gelatin zymography. Methods Mol Biol. 2012;878:121-35.

31. Padmanabhan P, Dmochowski R. Urinary incontinence in women: a comprehensive review of the pathophysiology, diagnosis and treatment. Minerva Ginecol. 2014;66:469-78.

32. Clobes A, DeLancey JOL, Morgan DM. Urethral circular smooth muscle in young and old women, American Journal of Obstetrics and Gynecology, 198 (2008).

33. Konala VBR, Mamidi MK, Bhonde R, Das AK, Pochampally R, Pal R. The current landscape of the mesenchymal stromal cell secretome: A new paradigm for cell-free regeneration. Cytotherapy. 2016;18:13-24.

34. Salgado AJ, Sousa JC, Costa BM, Pires AO, Mateus-Pinheiro A, Teixeira FG, Pinto L, Sousa N. Mesenchymal stem cells secretome as a modulator of the neurogenic niche: basic insights and therapeutic opportunities, Frontiers in Cellular Neuroscience, 9 (2015).

35. Song M, Heo J, Chun JY, Bae HS, Kang JW, Kang H, Cho YM, Kim SW, Shin DM, Choo MS. The Paracrine Effects of Mesenchymal Stem Cells Stimulate the Regeneration Capacity of Endogenous Stem Cells in the Repair of a Bladder-Outlet-Obstruction-Induced Overactive Bladder. Stem Cells Development. 2014;23:654-63.

36. Lin GT, Wang GF, Banie L, Ning HX, Shindel AW, Fandel TM, Lue TF, Lin CS. Treatment of stress urinary incontinence with adipose tissue-derived stem cells. Cytotherapy. 2010;12:88-95.

37. Wang Z, Wen Y, Li YH, Wei Y, Green M, Wani P, Zhang PB, Pera RR, Chen B. Smooth Muscle Precursor Cells Derived from Human Pluripotent Stem Cells for Treatment of Stress Urinary Incontinence. Stem Cells Development. 2016;25:453-61.

\section{Figures}



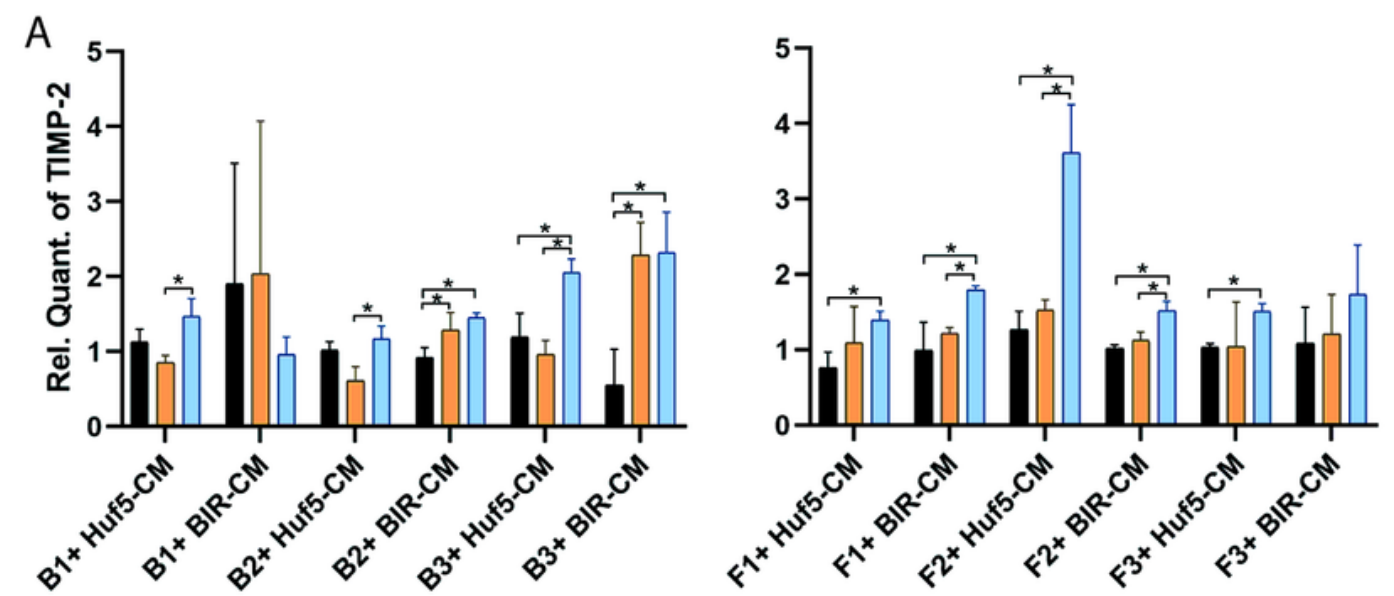

SMGS(control) $(n=3)$

CM p0 $(n=3)$

CM p3 $(n=3)$
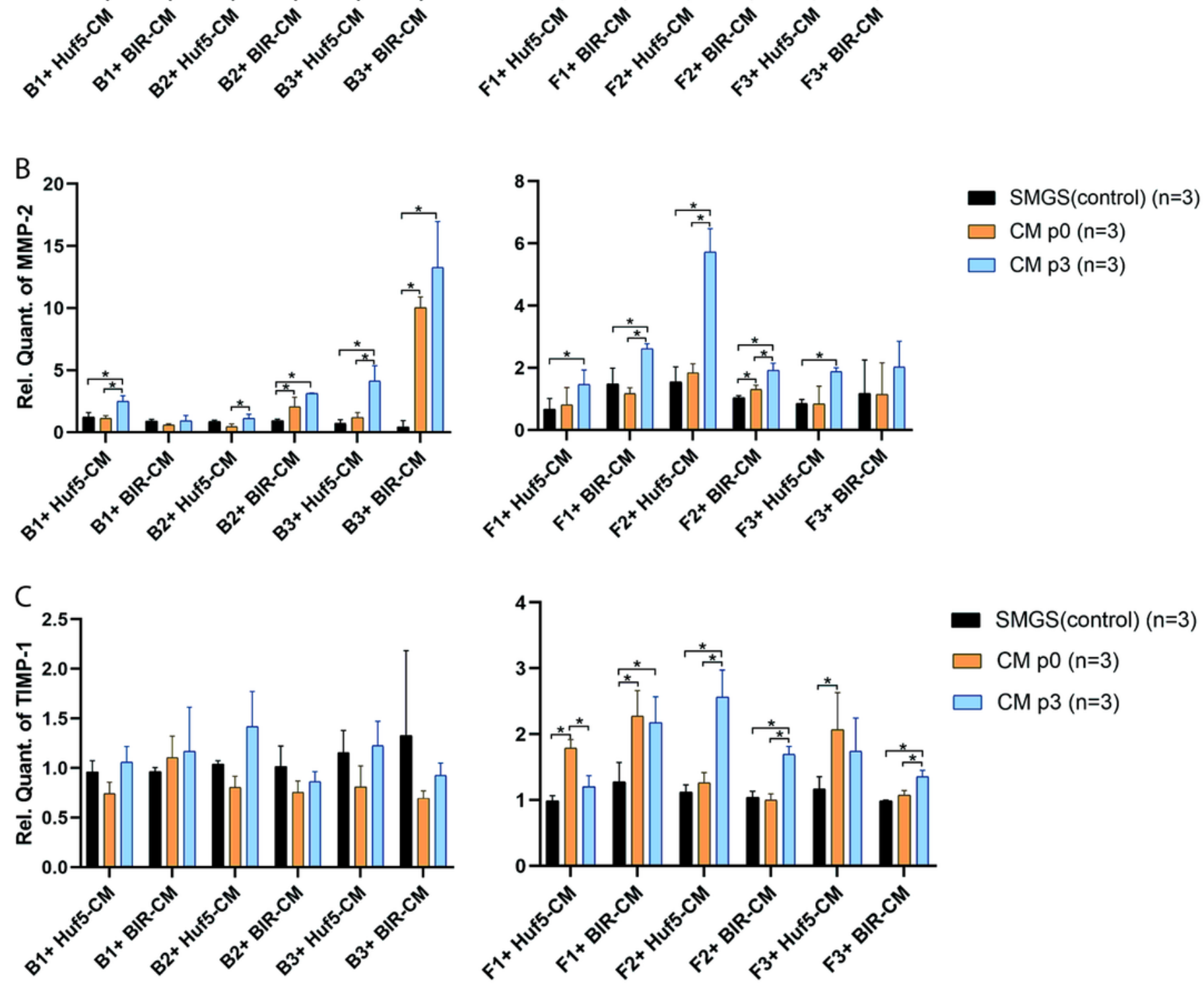

\section{Figure 1}

Gene expression of extracellular matrix (ECM) metabolism proteins in pSMC-CM-treated bSMCs and vaginal fibroblasts (A-C). Gene expression levels of TIMP-2 (A), MMP-2 (B), and TIMP-1 (C) were analyzed by quantitative real-time RT-PCR in bSMCs from three women (B1, B2, B3) and vaginal fibroblasts from three women $(F 1, F 2, F 3)$. Data shown represent the mean \pm SD from three independent experiments. Huf5-CM = conditioned medium from Huf5 iPSC-derived pSMCs; BIR-CM = conditioned medium from BIR 
iPSC-derived pSMCs; SMGS = bSMC or fibroblasts treated with SMGS only (controls). * = significant difference between groups $(p<0.05)$

A
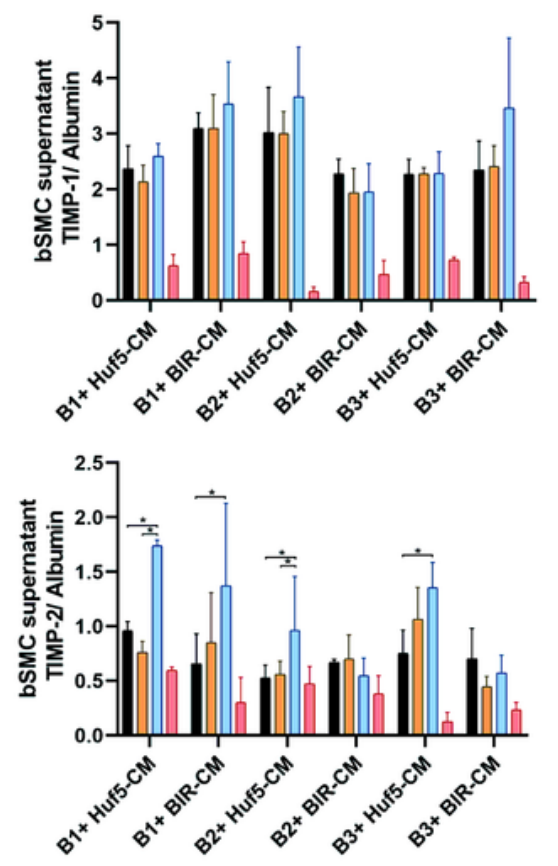

B
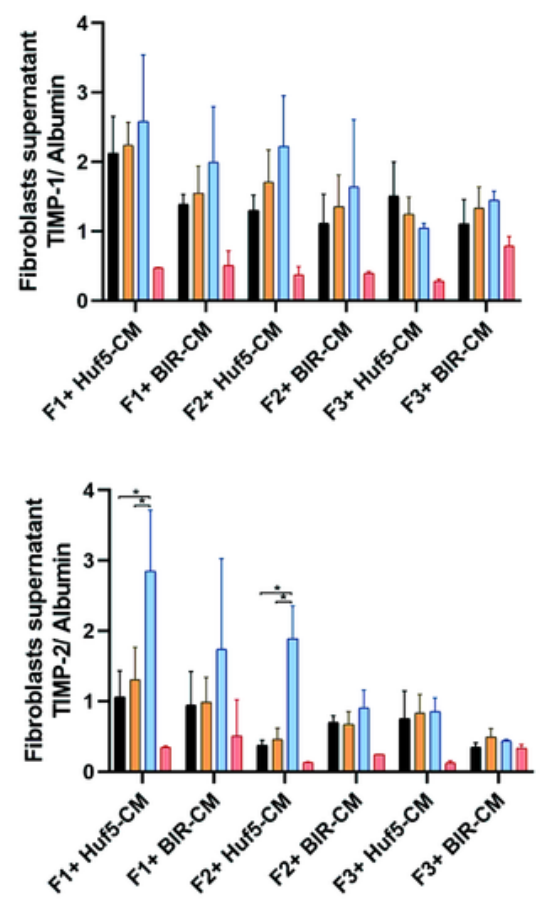

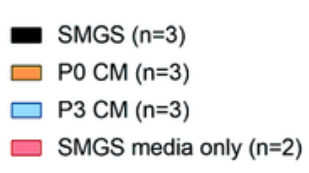

- $\operatorname{SMGS}(n=3)$

$\square$ PO CM (n=3)

$\square$ P3 CM (n=3)

$\square$ SMGS media only $(n=2)$

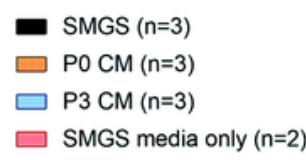

- $\operatorname{SMGS}(\mathrm{n}=3)$

$\square$ PO CM (n=3)

$\square$ P3 CM (n=3)

$\square$ SMGS media only ( $\mathrm{n}=2)$

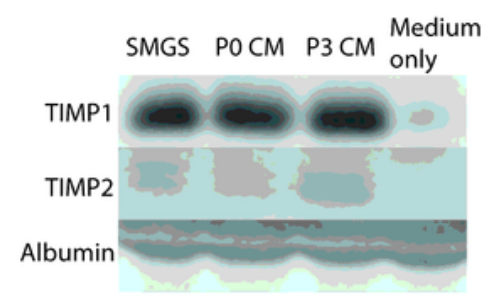

B1+ huf5 CM

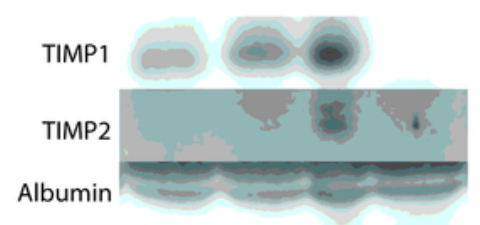

B2+ huf5 CM

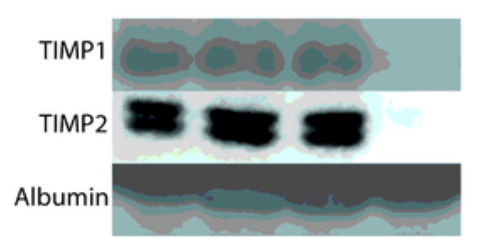

B3+ huf5 CM

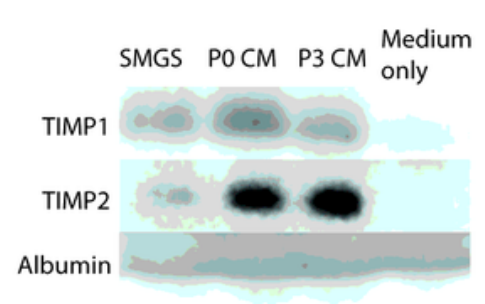

$\mathrm{F} 1+$ huf5 $\mathrm{CM}$

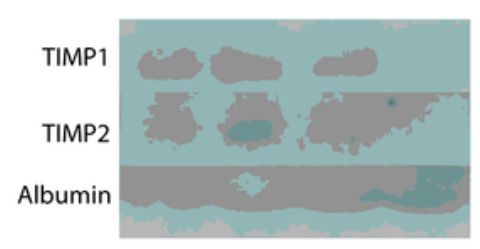

F2+ huf5 CM

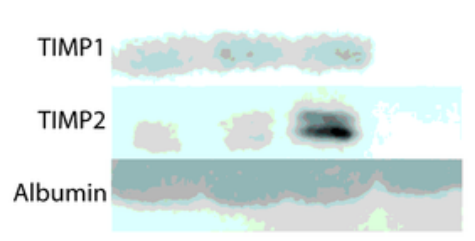

F3+ huf5 CM
$\mathrm{B} 1+\mathrm{BIRCM}$

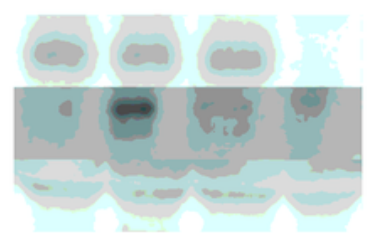

$\mathrm{B} 2+\mathrm{BIR} C \mathrm{M}$

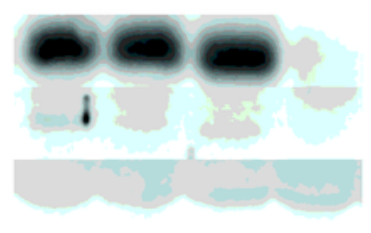

$\mathrm{B} 3+\mathrm{BIR} C \mathrm{M}$

SMGS POCM P3CM $\begin{aligned} & \text { Medium } \\ & \text { only }\end{aligned}$

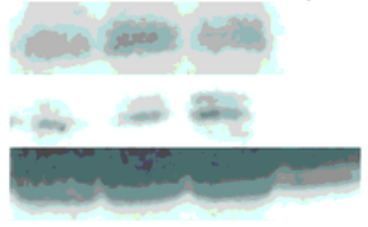

$\mathrm{F} 1+\mathrm{BIRCM}$

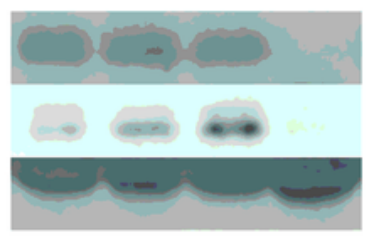

$\mathrm{F} 2+\mathrm{BIR} C \mathrm{M}$

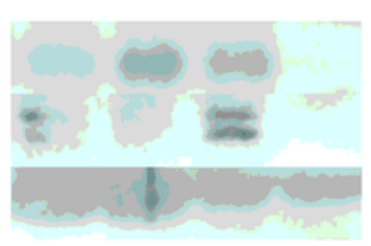

$\mathrm{F} 3+\mathrm{BIRCM}$

\section{Figure 2}

TIMP-1 and TIMP-2 protein expression in the supernatant of the bSMCs (A) and vaginal fibroblasts (B) treated with $\mathrm{pSMC}-\mathrm{CM}$. Data shown represent the mean \pm SD from three independent experiments. Huf5$\mathrm{CM}=$ conditioned medium from Huf5 iPSC-derived pSMCs; BIR-CM = conditioned medium from BIR iPSC- 
derived pSMCs; SMGS = supernatant from bSMC or vaginal fibroblasts treated with SMGS only; SMGS media only = concentrated SMGS media only. * = significant difference between groups $(p<0.05)$
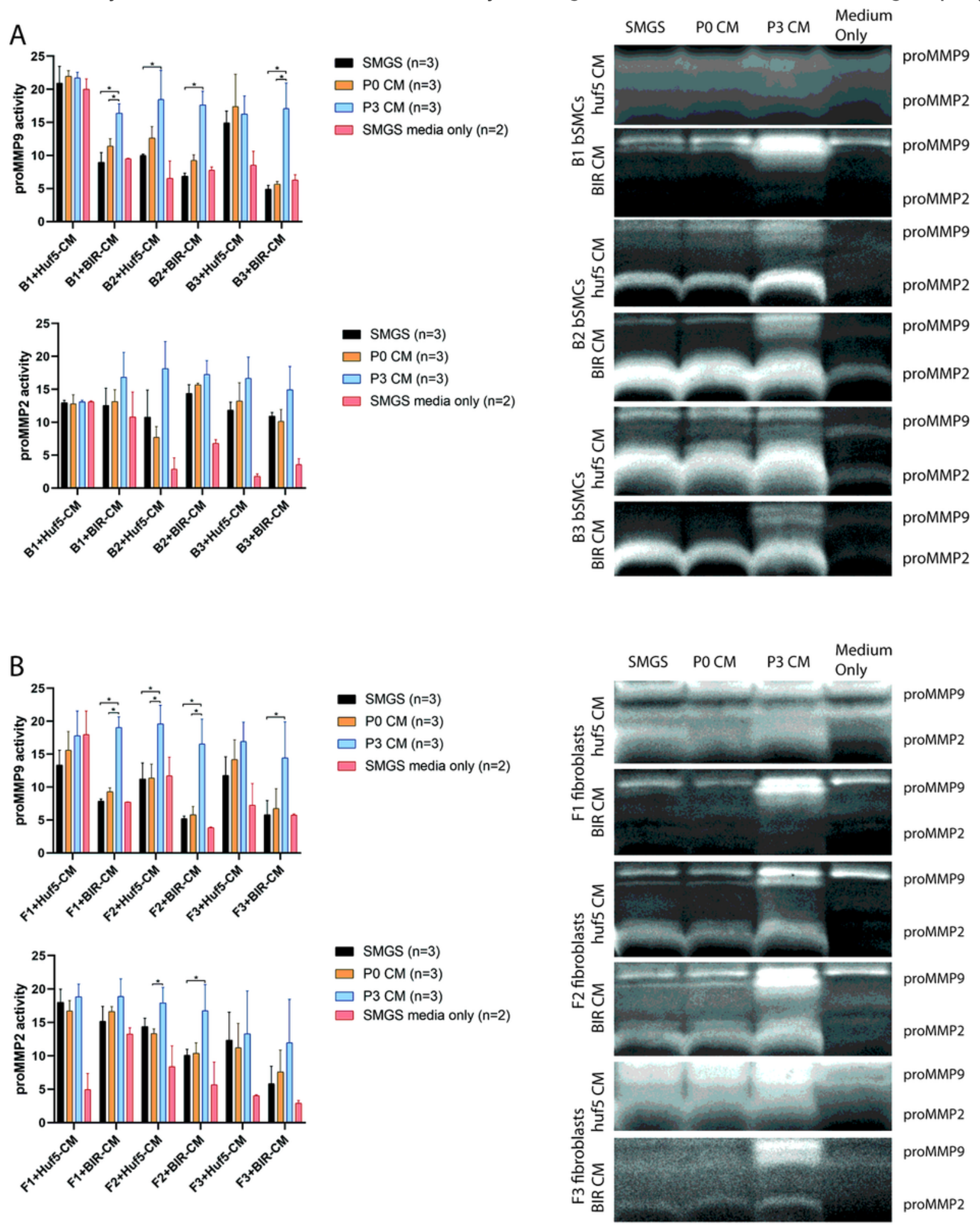

\section{Figure 3}

ECM protease activity of pSMC-CM-treated bSMCs (A) and vaginal fibroblasts (B). Zymographic evaluation of MMP activities in concentrated supernatant from bSMCs and vaginal fibroblasts treated with different conditioned medium. Zymograms are representative of three separate experiments. Data 
shown represent the mean \pm SD from three independent experiments. Huf $5 \mathrm{CM}=$ conditioned medium from Huf5 iPSC-derived pSMCs; BIR CM = conditioned medium from BIR iPSC-derived pSMCs; SMGS = supernatant from bSMC or fibroblasts treated with SMGS only; SMGS media only = concentrated SMGS media only. * = significant difference between groups $(p<0.05)$

A
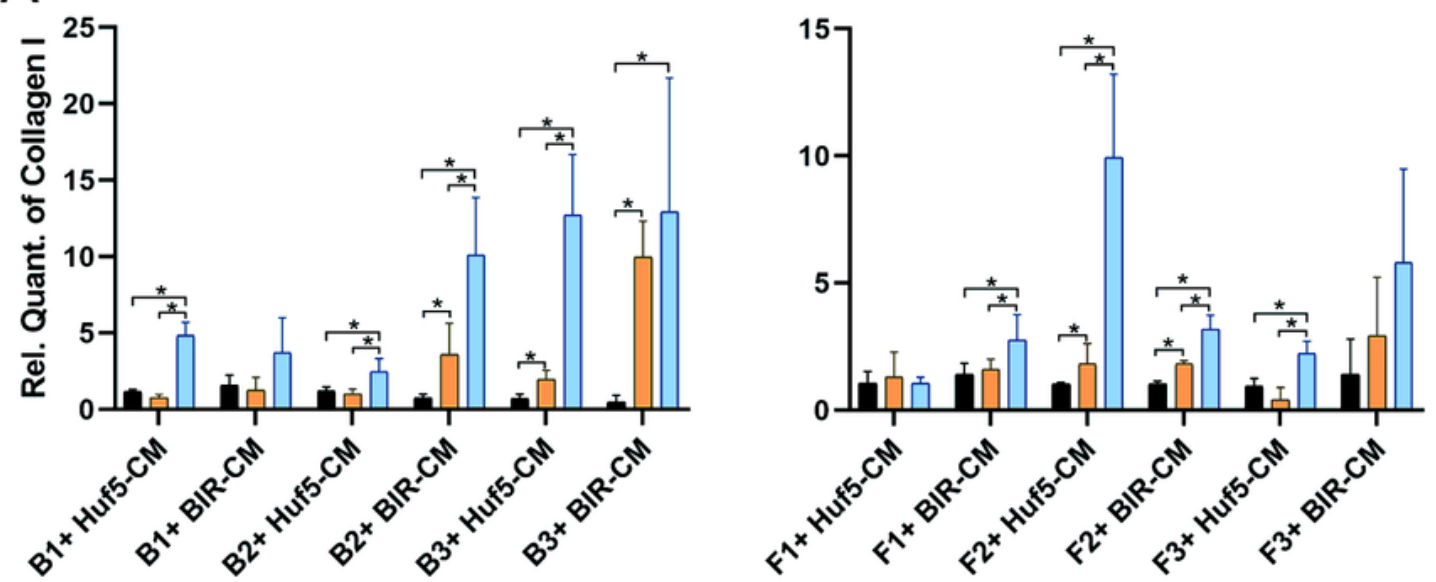

B
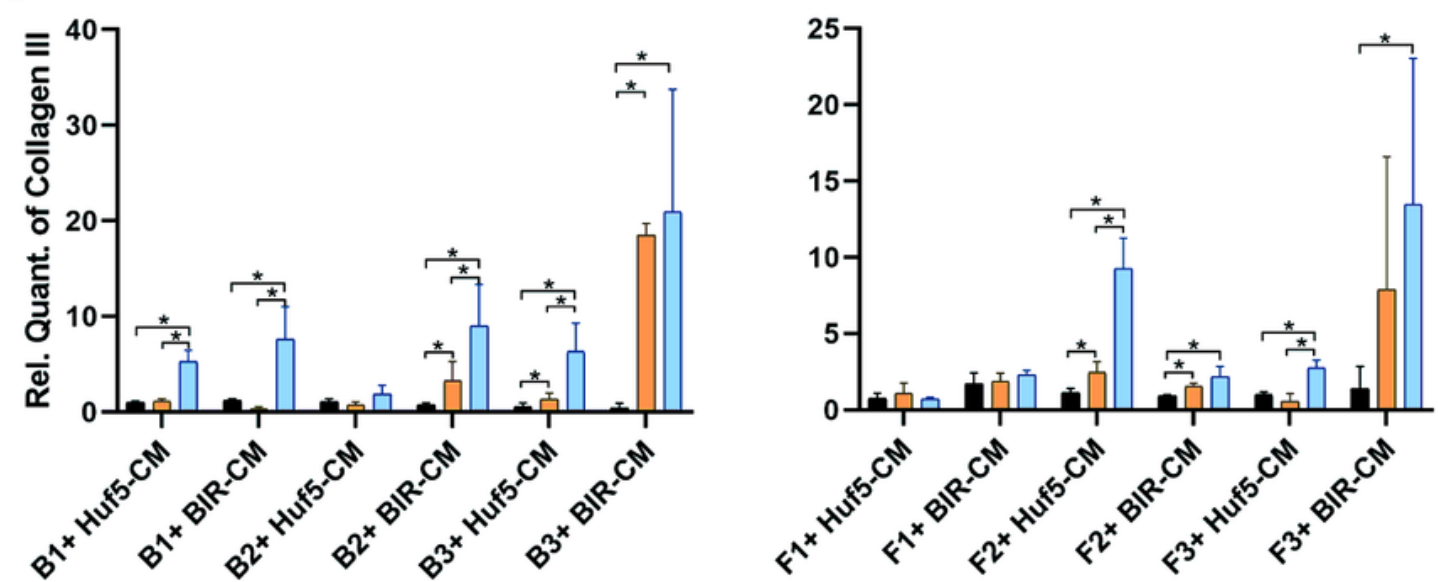

- SMGS(control) $(n=3)$

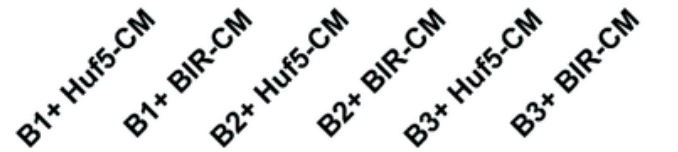

$\square$ CM p0 $(n=3)$

CM p3 $(n=3)$
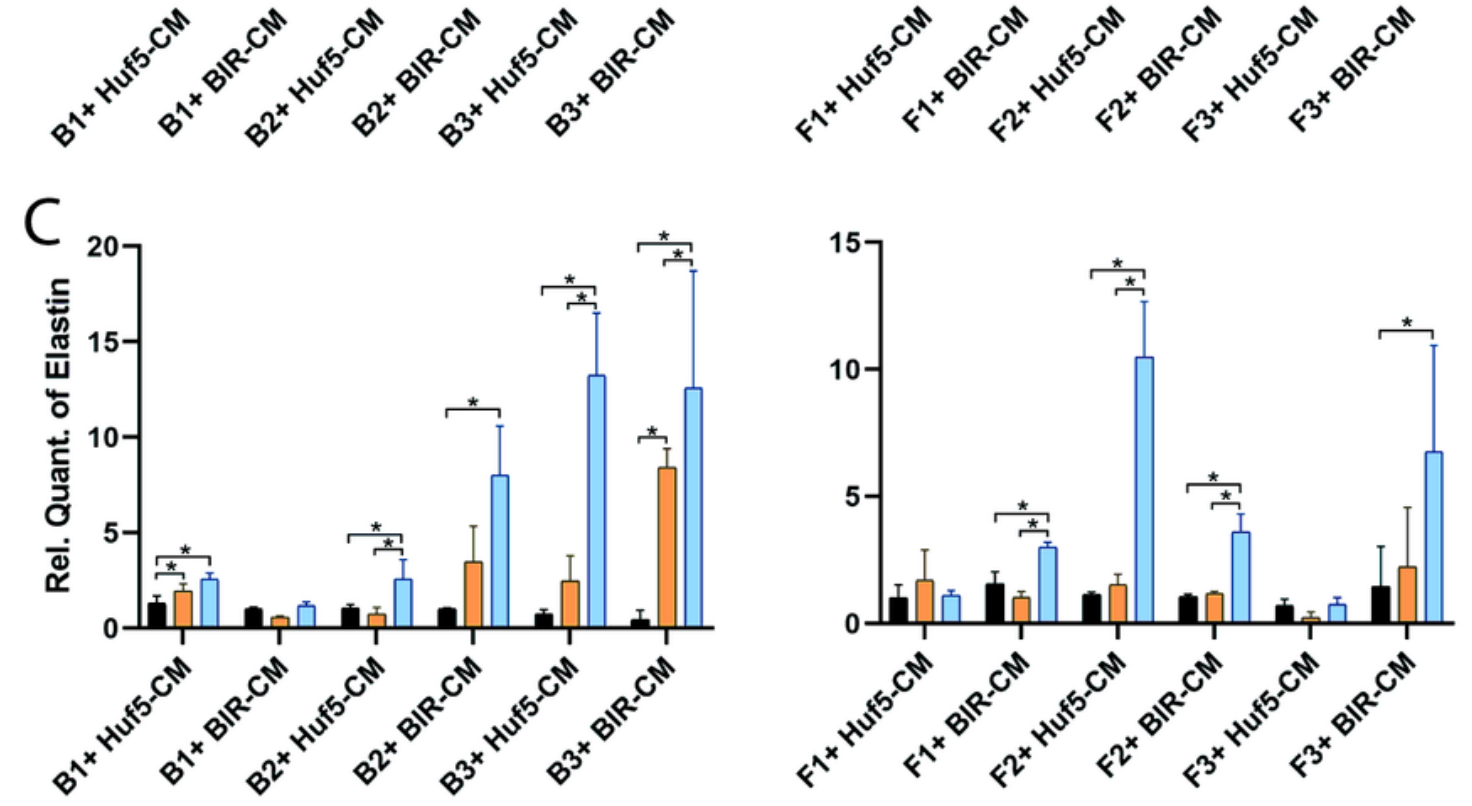

- SMGS(control) $(n=3)$

$\square$ CM p0 (n=3)

CM p3 $(n=3)$

- SMGS(control) $(\mathrm{n}=3)$

$\square$ CM p0 (n=3)

$\square \mathrm{CM} \mathrm{p3}(\mathrm{n}=3)$

Figure 4 
Gene expression of collagen I, collagen III and elastin in conditioned medium-treated bSMCs and vaginal fibroblasts $(A-C)$. Data shown represent the mean \pm SD from three independent experiments. Huf $5 \mathrm{CM}=$ conditioned medium from Huf 5 iPSC-derived pSMCs; BIR CM = conditioned medium from BIR iPSCderived pSMCs; $S M G S=$ bSMC or fibroblasts treated with SMGS only (controls). ${ }^{*}=$ significant difference between groups $(p<0.05)$

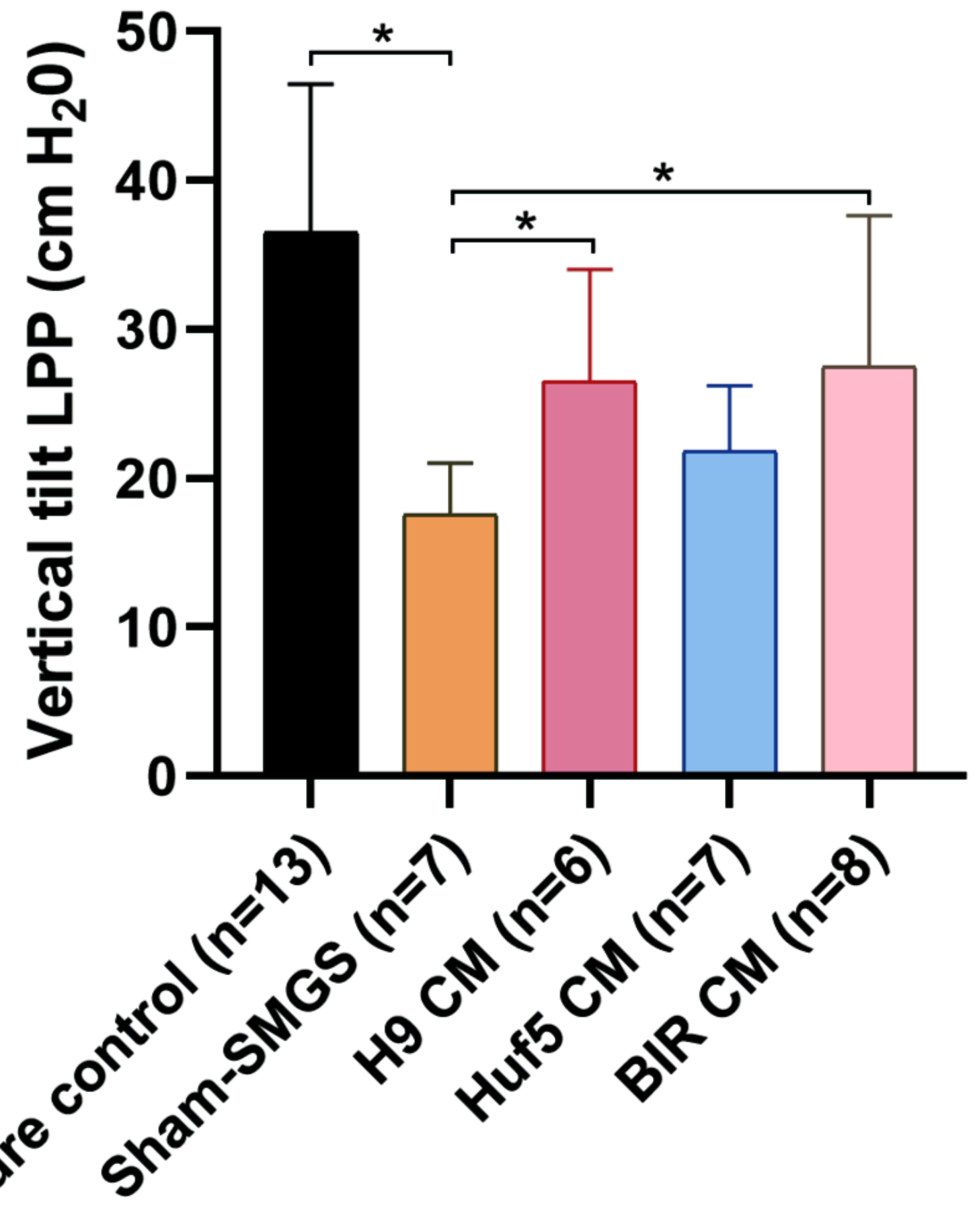

Figure 5 
Comparison of leak point pressure (LPP) values of SUI rats in different groups. Data shown represent the mean \pm SD. Pure control = rats with no surgery and no treatment; Sham SMGS = rats treated with concentrated SMGS only; $\mathrm{H} 9 \mathrm{CM}=$ rats treated with conditioned medium from H9 ESC-derived pSMC; Huf $5 \mathrm{CM}=$ rats treated with conditioned medium from Huf5 iPSC-derived pSMCs; BIR CM = rats treated with conditioned medium from Huf5 iPSC-derived pSMCs. * = significant difference between groups $(p<0.05)$

A

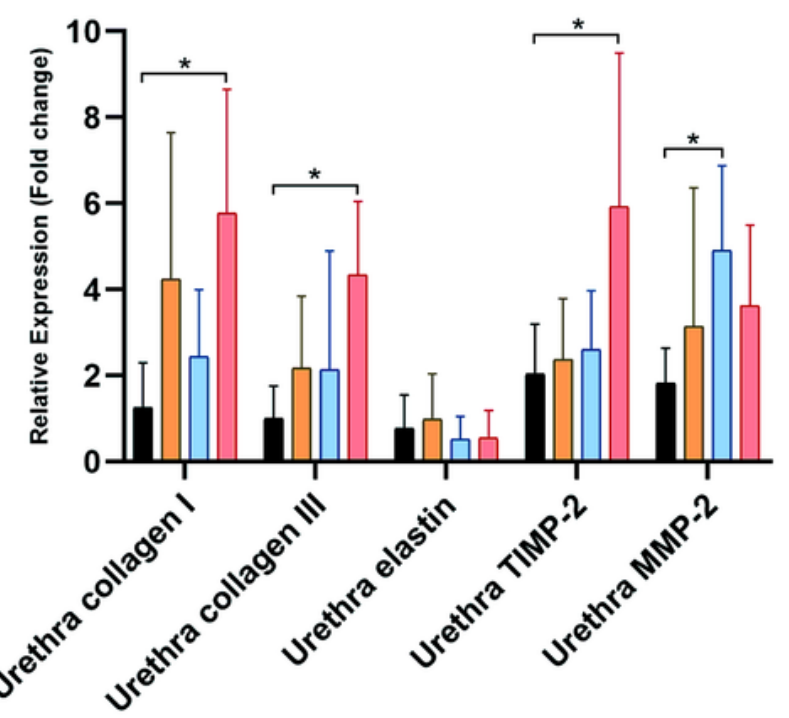

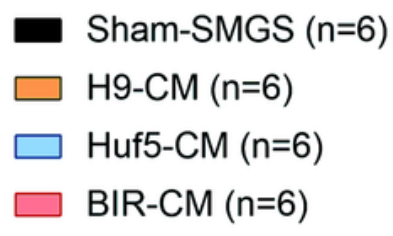

B
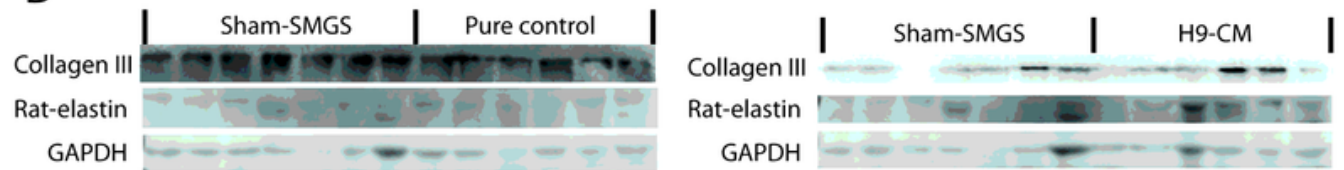

Collagen III

Rat-elastin GAPDH

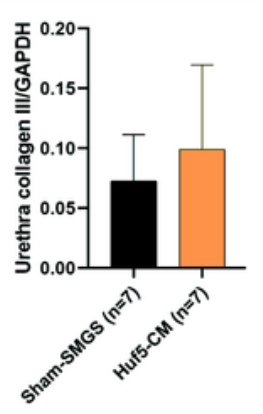

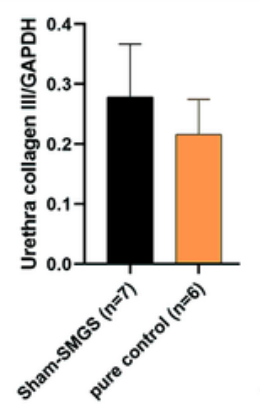
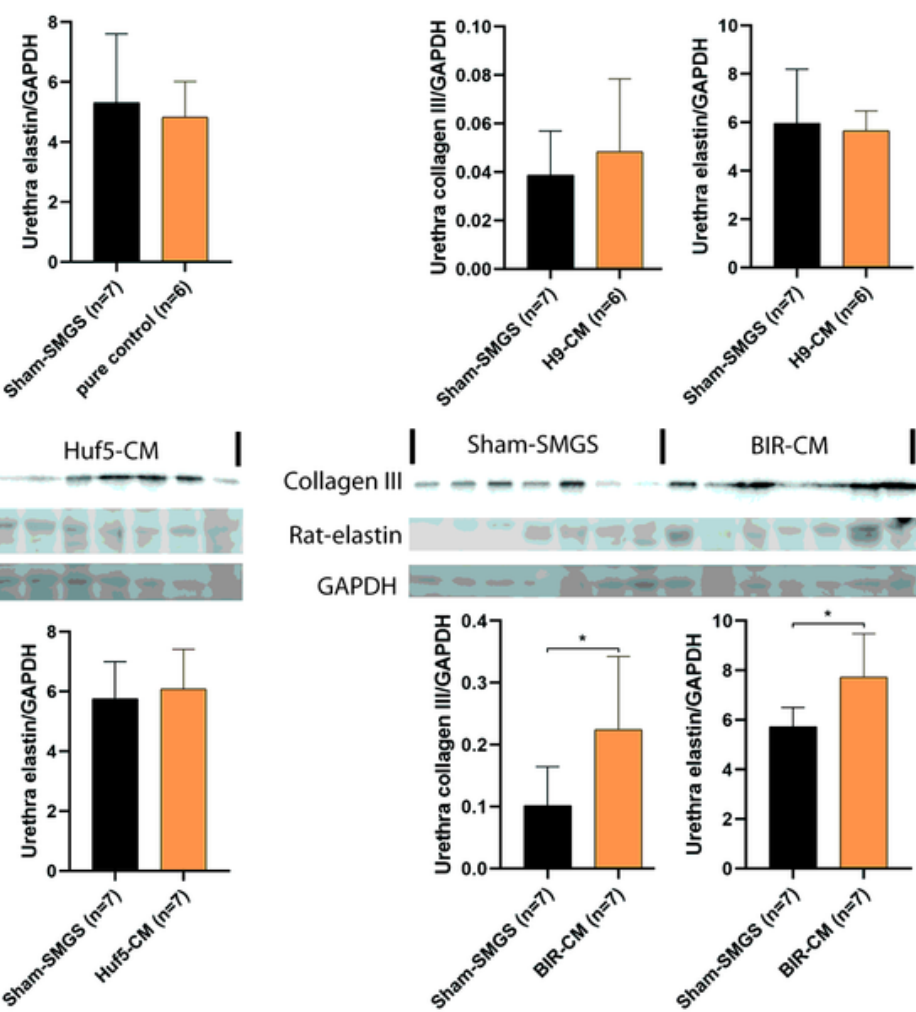

Sham-SMGS I Huf5-CM I
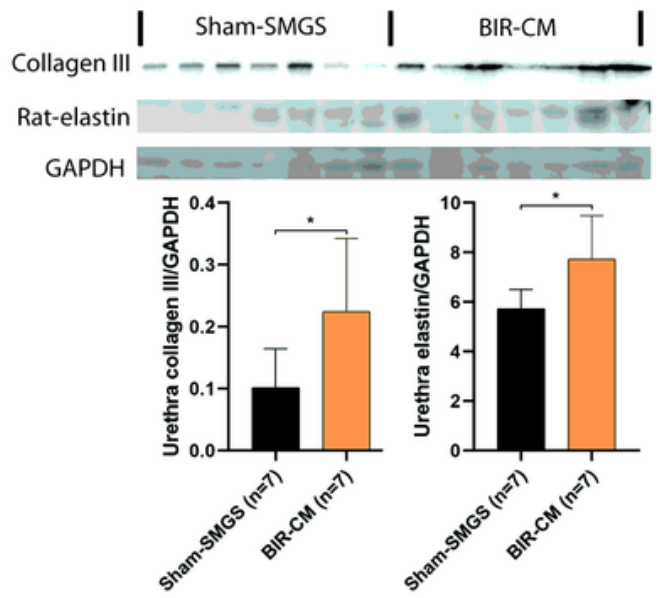

Figure 6 
Gene expression of rat-collagen I, rat-collagen III, rat-elastin, TIMP-2 and MMP-2 (A) and protein levels of rat-collagen III and rat-elastin in conditioned medium treated rat urethras for each group (B). Data shown represent the mean \pm SD. Pure control = rats with no surgery and no treatment; Sham SMGS = rats treated with concentrated SMGS only; $\mathrm{H} 9 \mathrm{CM}=$ rats treated with conditioned medium from H9 ESC-derived pSMC; Huf 5 CM = rats treated with conditioned medium from Huf5 iPSC-derived pSMCs; BIR CM = rats treated with conditioned medium from Huf 5 iPSC-derived pSMCs. * = significant difference between groups $(\mathrm{p}<0.05)$
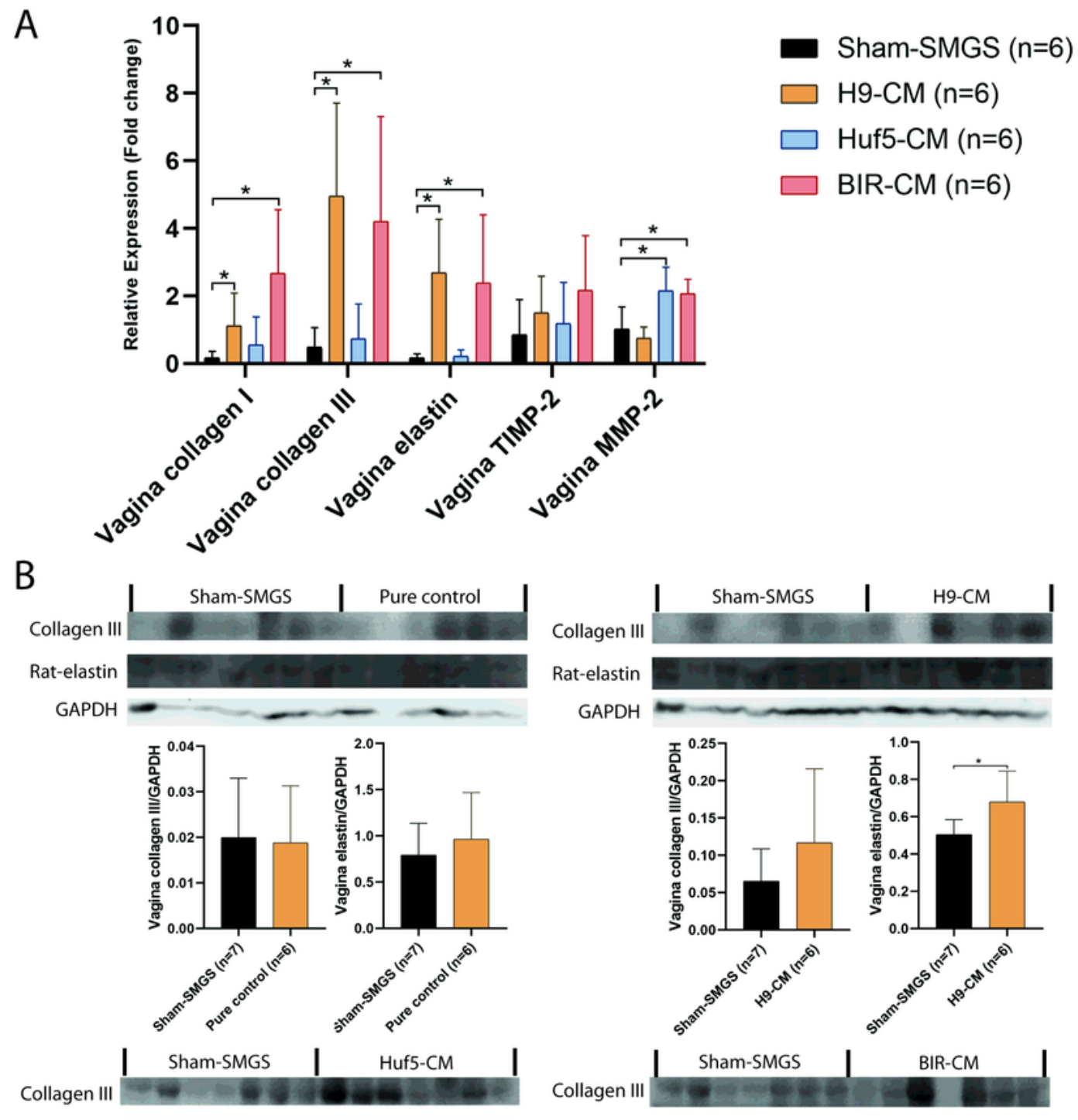

Rat-elastin
GAPDH

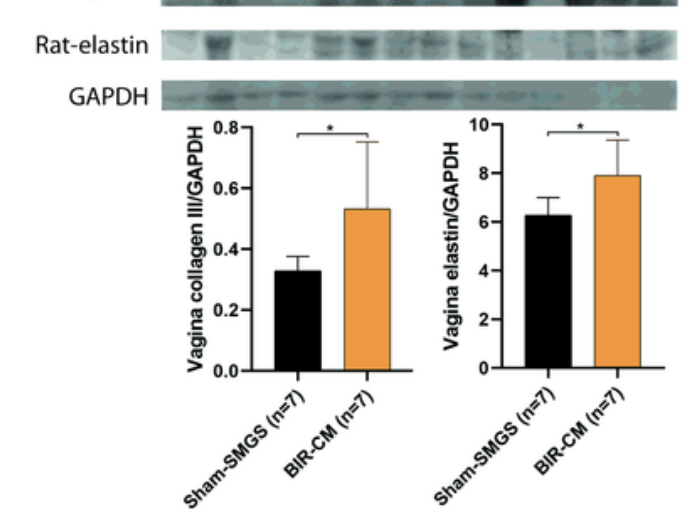




\section{Figure 7}

Gene expression of rat-collagen I, rat-collagen III, rat-elastin, TIMP-2 and MMP-2 (A) and protein levels of rat-collagen III and rat-elastin in conditioned medium treated rat vaginas (B). Data shown represent the mean \pm SD. Pure control = rats without surgery and no treatment; Sham SMGS = SUI rats treated with concentrated SMGS; $\mathrm{H} 9 \mathrm{CM}=\mathrm{SUI}$ rats treated with conditioned medium from H9 ESC-derived pSMC; Huf $5 \mathrm{CM}=\mathrm{SUl}$ rats treated with conditioned medium from Huf5 iPSC-derived pSMCs; BIR CM = SUI rats treated with conditioned medium from Huf5 iPSC-derived pSMCs. * = significant difference between groups $(p<0.05)$
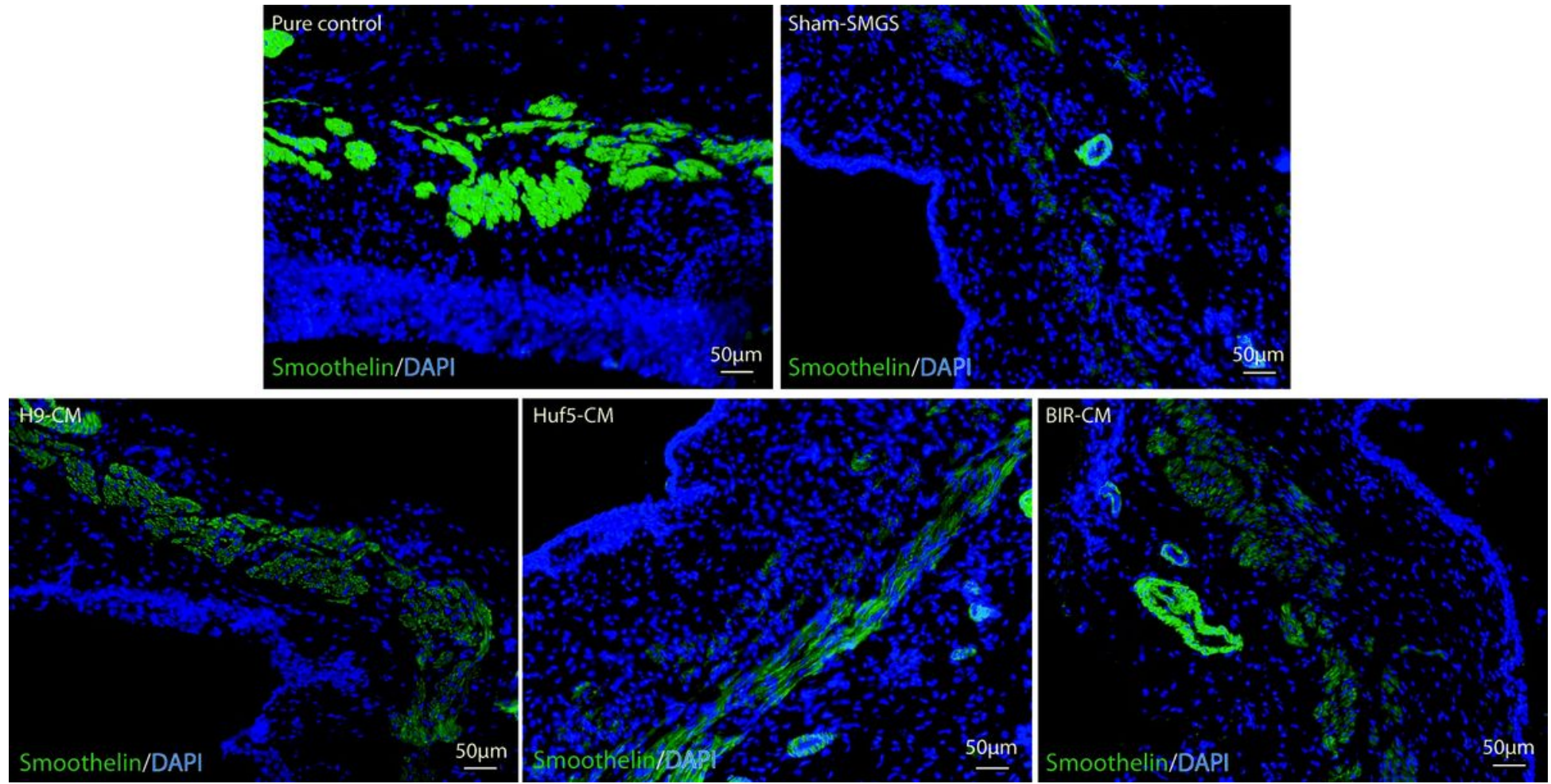

\section{Figure 8}

Effect of pSMC-CM on the muscle layer of the rat vagina wall. Representative images of cross-section of proximal vagina with smoothelin staining (green). Pure control = rats with no surgery and no treatment; Sham SMGS = SUI rats treated with concentrated SMGS only; $\mathrm{H} 9 \mathrm{CM}=\mathrm{SUI}$ rats treated with conditioned medium from $\mathrm{H} 9$ ESC-derived $\mathrm{pSMC}$; Huf5 $\mathrm{CM}$ = SUl rats treated with conditioned medium from Huf5 iPSC-derived pSMCs; BIR CM = SUI rats treated with conditioned medium from Huf5 iPSC-derived pSMCs. 